\title{
Landscape in the Economy of Conspicuous Consumptions
}

Hokky Situngkir

[hs@compsoc.bandungfe.net]

Dept. Computational Sociology

Bandung Fe Institute

\begin{abstract}
Psychological states side by side with the bounded rational expectations among social agents contributes to the pattern of consumptions in economic system. One of the psychological states are the envy - a tendency to emulate any gaps with other agents' properties. The evolutionary game theoretic works on conspicuous consumption are explored by growing the micro-view of economic agency in lattice-based populations, the landscape of consumptions. The emerged macro-view of multiple equilibria is shown in computational simulative demonstrations altogether with the spatial clustered agents based upon the emerged agents' economic profiles.
\end{abstract}

Keywords: conspicuous consumption, behavioral economics, agent-based simulations. 
Envy is ever joined with the comparing of a man's self; and where there is no comparison, no envy!

Thou shalt not covet thy neighbor's wife; thou shalt not covet thy neighbor's house, nor his field ... nor anything that is thy neighbor's. the $10^{\text {th }}$ rule in ten commandments

\section{Introduction}

There have been broad recent understandings on human economic behaviors outside economic discourses. Economic decisions are always becoming one of the most complex things in economic discussions. One of them is the way we choose our pattern of consumption. It has been a common understanding that the pattern of consumption is strongly related to the social and economic status. A lot of things are being marketed not only for the functionality or degree of necessity solely, but also related to the profile beneficiaries. Most luxurious stuffs are placed in the market as a sort of conspicuous consumption. They are being sold and bought for the need of good reputation, pecuniary strength and good name of buyers, or social leisure of having more than others. This is directly reflected in our daily urban and highly organized industrial society [19], from the type of cellular phones, dressings, style and brand, even place of lunch and recreation to the luxurious home living and daily vehicles.

While income is an important measure for social class, the pattern of consumption is the way to show other people conspicuously the represented social class. A good and unleashed description of the relatedness of between consumption is described in [18]. A lot of things are thus, bought in order to be shown to others. Furthermore, research surveys have also confirmed how social stratification correlated to the cultural consumption [2].

Nonetheless, those are economic phenomena since it has been directly related to the concavity of the supply and demand curves for particular products in the market, but yet, it is also related to a deep emotional and psychological trait of human species, envy. This is related an interesting field related to behavior economics. Inequality has been understood to be one of source of unhappiness among people (cf. [7]). Furthermore, the interesting relations between inequality with the state of well-being or happiness are related to the emotional states of human being. As it has been noted in [13], emotions serves an adaptive role in helping organisms deal with key survival issues posed by the environment. Emotional based decision making among economic agents might have been one of explanation to the deviation of rationality in the sense of conventional understanding (cf. [10]), beside the realizations on the social boundedness on which rational choices must be taken ([16] \& [9]).

Recent economic discussions have introduced a lot of interesting discourses related to this. The evolutionary game theory as harmonious mixtures between biological studies, economic behavior, and mathematical theories on games [17] has outlined some applications into the recent problems in economics [5]. One of interesting points are related to the formalizations of the social pattern regarding to the game of the conspicuous consumption [4]. The discourse of the evolutionary game theoretic analysis on economic issues, the emotional based economic decision making as well as the boundedly rational agent based model employment in computational analysis are those becoming main issues motivated the paper. 
The paper is begun with the discussions related to the overview of the mathematical models related to the conspicuous consumptions. M ost development of the model has been explored in the fashion of analytically evolutionary game theoretic models. Discussions are continued to the implementation of the models for wider theoretical explorations by incorporating the acquisitions of computational simulations. By the end of the paper, some demonstrations of the toy model are presented with some outlines to some conjectures in further development.

\section{Overview of the Model}

The presentation of the paper shows lattice-based populations as landscapes reflecting the allocations of ordinary and conspicuous expenses by economic agents. As described in [8], the lattice based populations, or more generally the neighborhood structure usually "tends to favor the longterm co-existence of strategies which would not co-exist in well-mixed populations". This is the dynamics as once introduced in [1,6] and thus implemented in cultural dissemination model [12], and also used as model to discover some dynamical characteristics in corruption [14]. The platform is particular kinds of agent based model [11] in which we utilize as computational experiments media. Another similar previous work related to advertising could also worth for mention [15].

Imagine a landscape where people are represented on lattices and grids. Each agent is given the same amount of money, and it is on their decision to allocate an $x=(0,1]$ amount of the money for ordinary necessities. While the savings and investments are neglected, the $1-x$ fraction of the money is thus allocated for luxurious expenditures. The later expense is thus becoming the source of envy among economic agents, things that influence their apprehension on their surroundings and thus give impact to their decision making. Thus, the expected pay off on each round of the game thus depends on each agent's ordinary consumption $(u)$ and her allocation of for the conspicuous consumption $(U)$,

$\varphi=U+c u$

where $c \geq 0$ denotes the constant marginal rate substitution. The ordinary consumption can be stated as a concave utility function,

$c u=c \ln x$,

and as $x \rightarrow 0$ we have $\varphi \rightarrow-\infty$ reflecting the importance of the ordinary consumption, while the utility function due to ordinary expenses would be $c u \in(-\infty, 0]$ - a fact that no one will completely neglects the ordinary consumption expect for the limiting case of $c=0$.

The main of the focus in the game is thus the conspicuous consumption: how much is the fraction needed to satisfy the social effect of envy when other surrounding people have more (or less) fraction for the conspicuous expenses. An evolutionary modeling of this has been analytically analyzed by the calculating the gradient dynamics in the fashion of cumulative distribution of function [4].

Our approach in the paper is related to the implementation of the models showing the economic behavior as pointed out in [19] as consumptions of the excellent goods that is socially related to the evidence of wealth. In this game, if an economic agent compare her allocated expenses for excellent goods is bigger than other, she would be in the psychological state of envy - a thing that motivates her to allocate bigger consumption if they interact again in the future. This envy however must be constrained due to her allocation of ordinary consumption which is a necessity and cannot be 
nullified. Here, when an agent allocates a fraction of $x$ to the ordinary consumption (thus allocate a fraction of $(1-x)$ for conspicuous one) and find out that other agent that interacts with her pay the fraction of $y<x$ for the same respective expenditures (thus $(1-y)$ for excellent goods), then she will have disutility in the proportion of

$r(x, y)=\min \{0,(y-x)\}$

In the analytical model as proposed by [4], the payoff can be written,

$U(x, D)=\int_{0}^{1} r(x, y) d D(y)=\int_{0}^{x}(y-x) d D(y)=-\int_{0}^{x} D(y) d y$

where $D(y)$ is the cumulative distribution function of other's decision on the fraction to the ordinary consumption and give us a consequence to the total payoff of an agent,

$\varphi(x, D)=c \ln x-\int_{0}^{x} D(y) d y$.

It is easy to see that that in the limiting case $\int_{0}^{x} D(y) d y=0$, a corresponding agent that allocates sufficiently small amount of $x$ for ordinary consumption gets the maximum payoff related to her envy to others. The gradient dynamics, on which agents adjust the value of $x$ is.

$\varphi_{x} @ \frac{\partial \varphi}{\partial x}=\frac{c}{x}-D(x)$

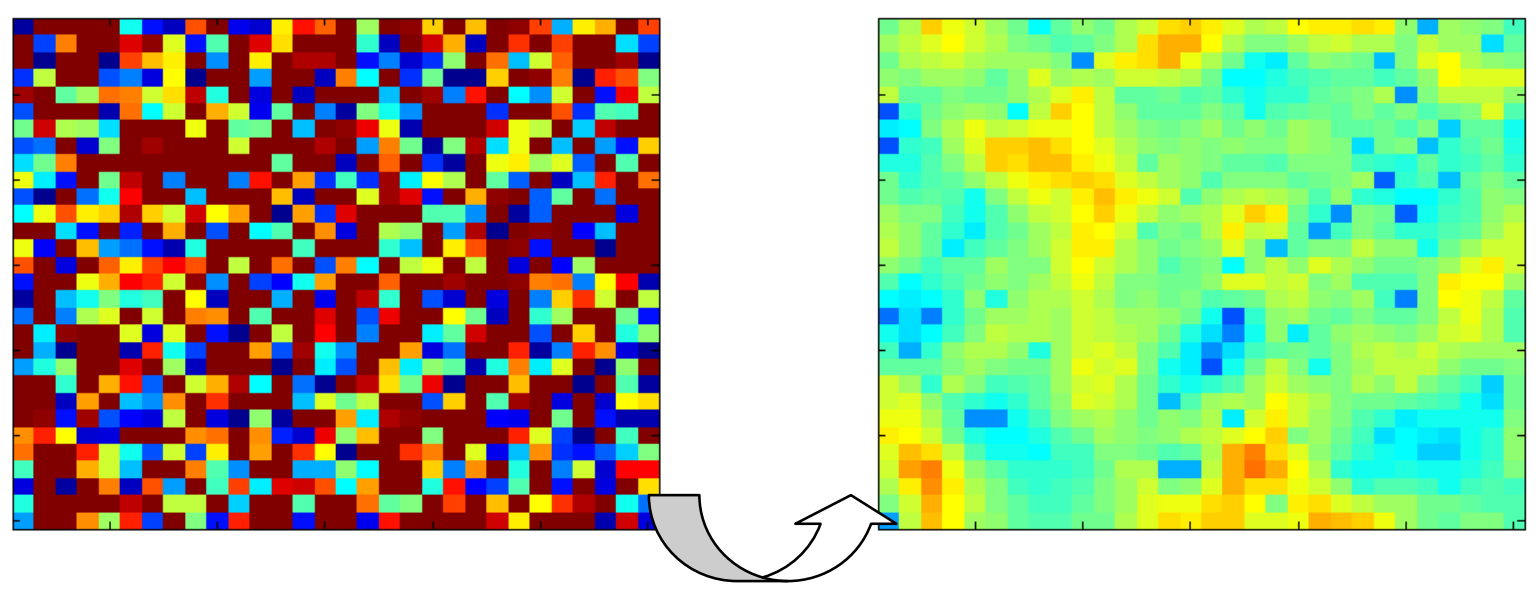

Figure 1. The landscape of allocation for ordinary consumption after the rule of envy is implemented for $\mathrm{T}=250$ rounds

\section{Experiments with spatially-bounded agents}

A numerical simulations is conducted in [5] in order to see the possible stable equilibria of the problems by a discretization of equation (5) in such a way, 
$\varphi=c \ln x-\sum_{i=1}^{N} F_{i} \Delta x=c \ln x-\frac{1}{N} \sum_{i=1}^{N} F_{i}$

With the gradient as analytically shown in eq. (6),

$\varphi_{n}=\frac{c}{x_{n}}-F_{n}$

where $F_{i}$ denotes the cumulative distribution function obtained from the numerical integration of the probability measure $f_{i}$ in the $i$-th interval among

$$
F_{i}=\sum_{k=1}^{N} f_{i} \Delta x
$$

as $f_{i}$ is the average density of $M$ number consumers with strategies divided in $\Delta x$ equal $N$ number of intervals, as to $\Delta x=\frac{1}{N}$. Consequently, we have $F\left(x_{0}\right)=0$ and $F\left(x_{N}\right)=1$, a sort of rank among whole agents - from which the name rank dependent consumption came from.

When the focus of our attention is the multiple equilibria, this approach has given interesting results. Yet, another perspective can also be offered related to the economic agent's boundedness related to the conspicuous consumption. In reality, the people are "trapped" in social surroundings in which envy and vanity is sourced upon cognitively. People do not have to compare his expenditures with those he would only "meet" on television, but people that are socially related to them.

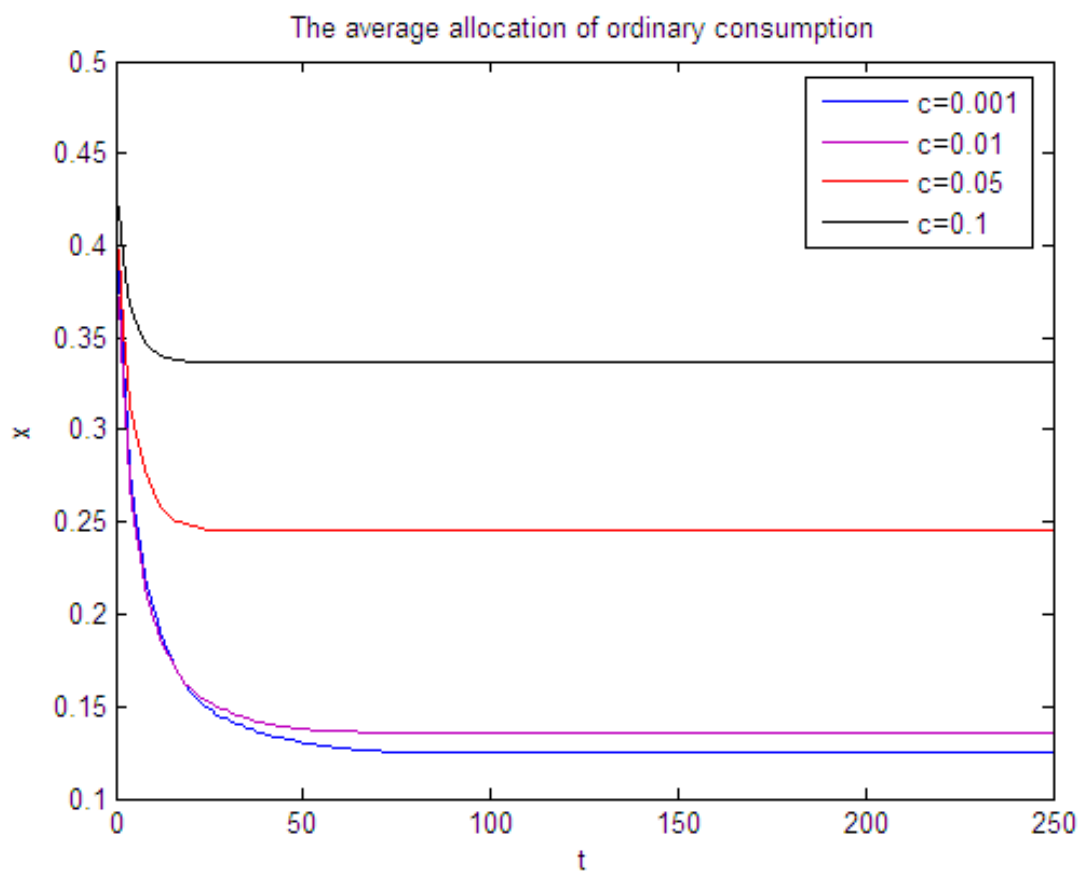

Figure 2. Different average equilibra at different values of $c$. 
Thus, we transform the discretization as suggested in eq. (7) into representation of agents placed in cells or square lattices located at a two-dimensional virtual world of $i, j=1, \ldots, L$ while the global view is more like a torus: the lowest two-dimensional grids are pasted together with the highest, and the right grids with the left one. The myopic agents evaluate their payoffs in the Moore neighborhood (with connections between nearest and next-nearest neighbors, thus each agent has total neighbors, $\left.\left[N_{v}\right]=8\right)$. In each time step $t=1,2, \ldots, T$, every agent evaluates her payoff from the set of strategies $x \in(0, \infty]$ and as an adaptation of eq. (1), (3) and (4), we can write the pay off as,

$$
\varphi(i, j, t)=c \ln [x(i, j, t)]+\frac{1}{\lambda} \sum_{N_{v}}\left[x(i, j, t)-x\left(i_{N_{v}}, j_{N_{v}} t\right)\right]
$$

where $x_{N_{v}}$ is the decision of neighbors for ordinary consumption and $\lambda$ denotes the number of neighbors, indexed as $N_{v}=\{1,2, \ldots, 8\}$, that has made the respective agent envious, $x(t)<y_{N_{v}}(t)$.

On any

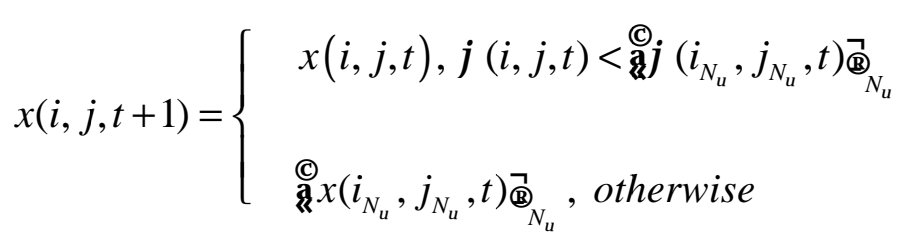

where ${ }_{x}\left(i_{N_{v}}, j_{N_{v}}, t\right)$ is the average of the average decision of neighbors for ordinary consumption. Thus, an agent would stay with the same fraction of ordinary consumption if her payoff is larger than the average payoff of her neighbors and change her decision into the average of her neighbors' decisions for ordinary consumption otherwise.

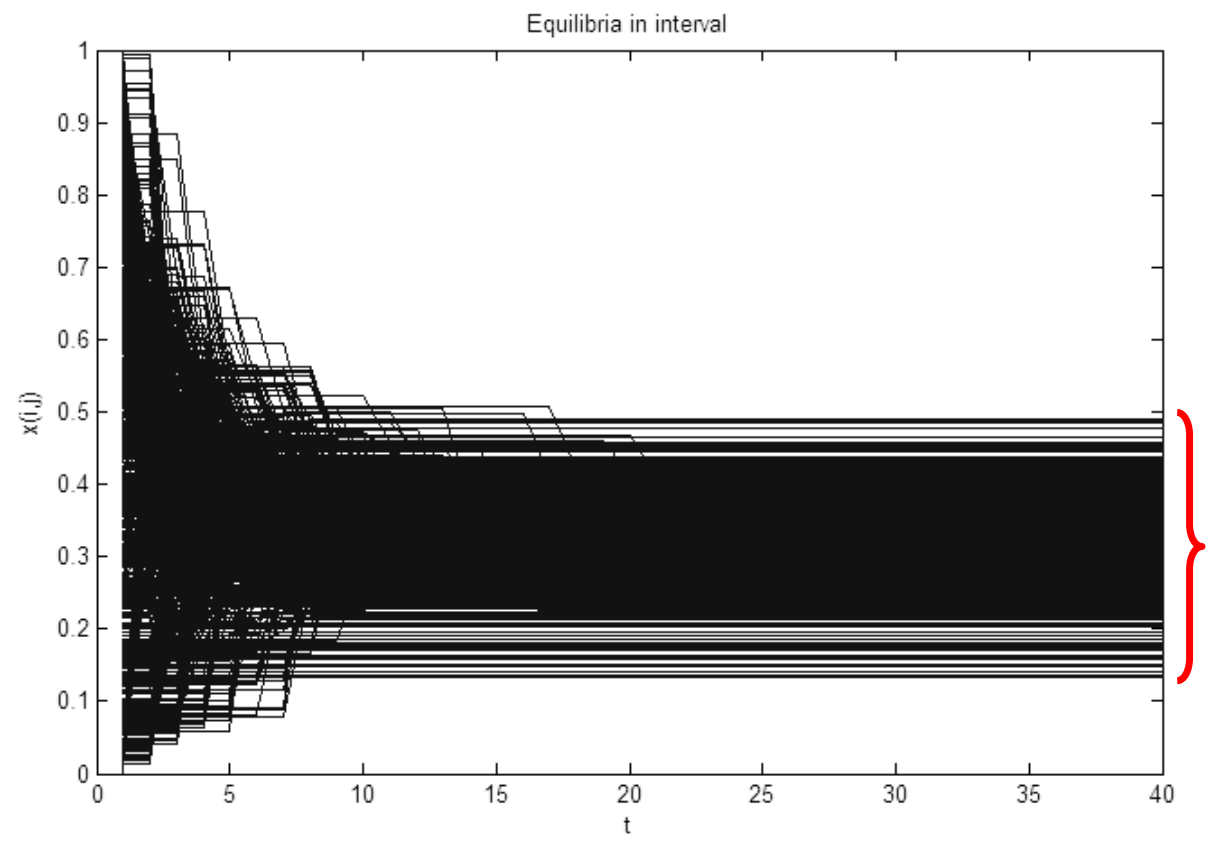

Figure 3. Agents' equilibrium points for $c=0.1$ 


\section{Computational Experiments}

From the model, we can do computational experiments that would reveal the unique equilibria among agents due to their allocations of ordinary and conspicuous consumptions. Our simulation is conducted in square lattices of $L=30$ for $T=250$ rounds of games with variable $c=.1$. The simulative process is shown in figure 1: the initial random condition of allocation to ordinary consumption and thus after rounds of simulation stay at equilibria when agents do not change their strategies for more. The not-changing states reflect the equilibrium and can be seen on the constant average allocation of ordinary consumption as shown in figure 2 for various value of marginal rate substitution $(c)$. It is clear that the higher the value of $c$, the more fraction of income to ordinary consumption should be allocated.

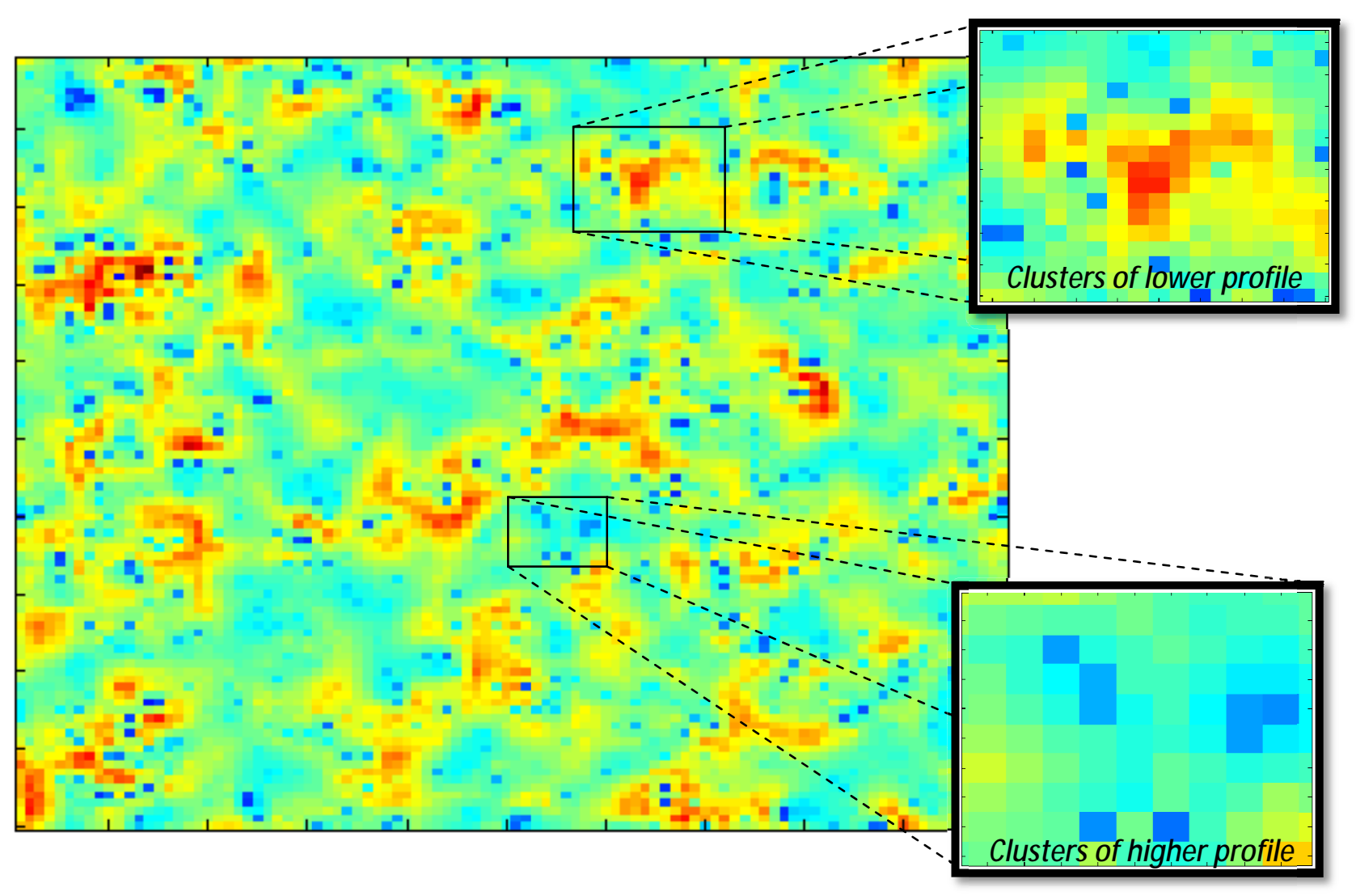

Figure 4. The emergence of social class: the allocation for ordinary consumption shows the high, low profile agents.

One of interesting thing that we can learn from our simulation is the emergence of spatially located social classes. It is worth to note that the average value as shown in figure 2 came from a sort of intervals in which agents stop changing their allocation for ordinary consumption. One of the results for $c=0.1$ is shown in figure 3. However, it is interesting to see that most of agents are finding their equilibrium point near the value of the average as some less agents are keeping their status as the higher and lower profile agents. Thus, the equilibria is shown to be in a kind of interval.

Interestingly, this can also be visualized as the clustered agents spatially as shown in figure 4 . At the end of rounds of simulation, it is obvious that there are some clustered agents to be in the state of higher allocation for ordinary consumption (i.e.: lower profile agents), lower one (i.e.: higher profile agents), while most of agents are in the state of medium class of ordinary consumption. This has become a unique property that we could have gained from the computational agent-based model 
from the model as introduced in eq. (5) and (6). From the big picture, there has been an emergence of economic classes based due to their profile in their allocation to ordinary and luxurious consumption in the state of multiple equilibria: there are "islands" representing the higher profile and lower profile agents among the sea of average profile persons.

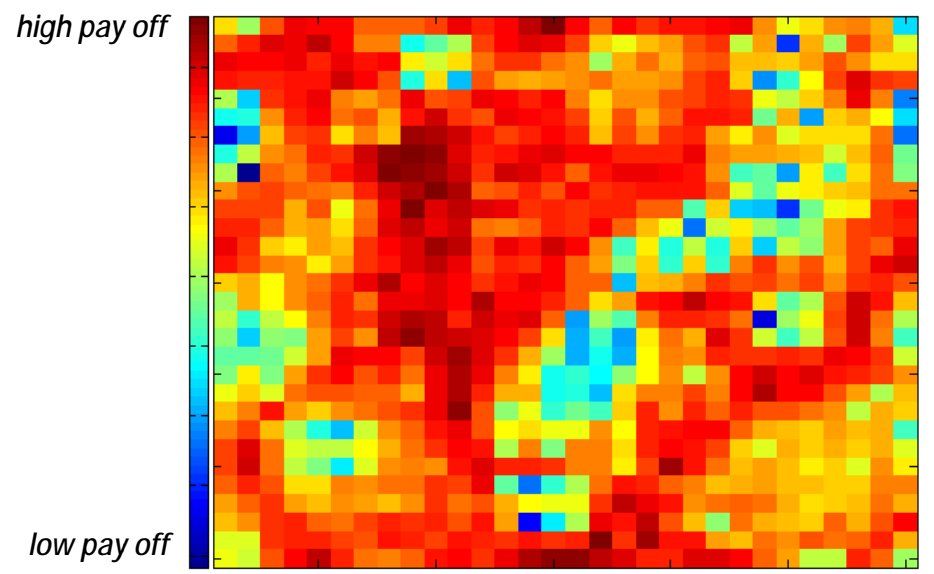

Figure 5. The landscape of payoff after $\mathrm{T}=250$ rounds: the darker the red cells are filled with agents whose higher accumulated payoffs.

From our simulation, we could also observe some interesting aspects spatially. Some agents are lucky enough that they have already close enough to the equilibria when the random initializations are set or at least they have neighbors with such allocations of ordinary consumption. Those have accumulated payoff relatively higher than the others. Figure 5 shows this in our landscape of consumptions. Obviously, the emergent spatial patterns are shown here. In the figure, darker red locations filled with such lucky agents. Rounds by rounds in our simulations they accumulate payoffs in such ways emerging the spatially clustered agents whose relatively higher payoffs at the end of our simulations. Those agents are clustered one another and to the clustered agents whose lower accumulated payoffs there would be such gradation. This reflects the diffusions of strategies throughout the spatial landscape related to the optimum and best fraction of ordinary consumption.

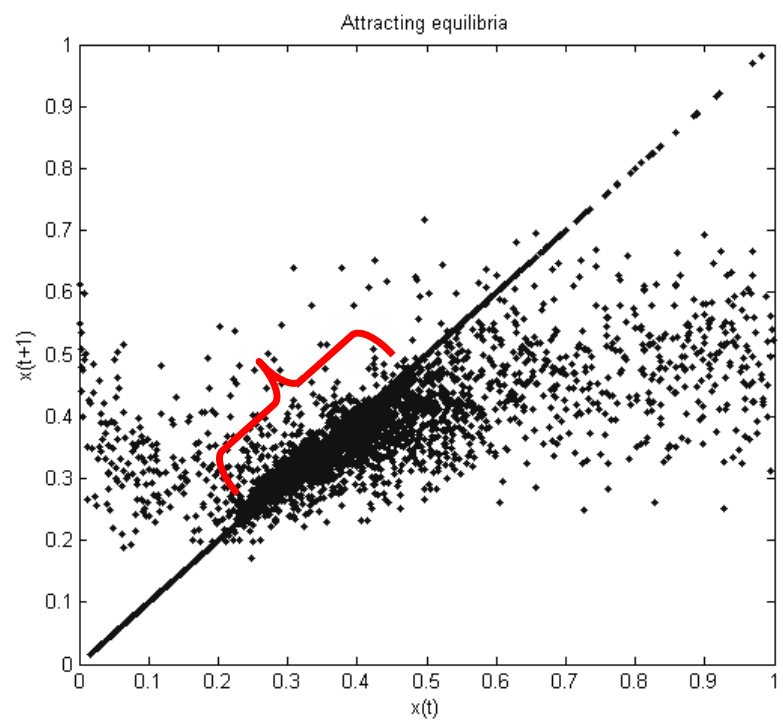

Figure 6. The equilibrium points as the attractors based on envy among agents throughout the computational simulation. The interval of the equilibria is shown. 
However, the process of the diffusions along the rounds of our computational simulations can be seen vaguely in the phase map corresponding to the mapping of $x(t) \rightarrow x(t+1)$. This is shown in the figure 6.The random initialization has been attracted to the intervals of equilibria. This is a sort of attractor of envy. Whatever the initial strategies, they will always be attracted to the intervals as shown in figure 3, and the equilibrium strategy decided by agents are thus related directly to their respective spatial positions i.e.: in what kind of neighborhood she is placed upon. Thus we have demonstrated that the evolutionary processes do not only depend upon the strategies that are being used in the games but also the respective positions of the strategic occupants.

\section{Concluding Remarks}

We have shown the discussions incorporating the computational agent based models of the game on allocating ordinary and conspicuous consumptions among economic agents. While the task came from one of the proxy of the evolutionary (economics) game theory, the presentation is based on an implemented fashion of computational simulations. The latter has been enriched the works on the boundedness of agents due to the myopic evaluations of agents on their neighborhood and some spatially emerged facts while macro-view equilibria have been reached over rounds of simulations. An interesting result is shown related to the varying, yet clustered populations and an attractor of envy on the landscape of allocations between ordinary and conspicuous consumption. While the size of the allocation for conspicuous consumption is related to the economic profile of agents, it is also visually shown in 2-dimensional lattices the emergence of higher, lower, as well as medium classes of economic profiles.

While in general the model depicts how emotional state - in this case, envy - could become a source of the concavity of the demand function, the promising future works could be conjectured to see how evolutionary game theory can be explored for other factors and variables used by agents, e.g. the development of economic discourse based on psychological behaviors, evolutionary paradigms on multiple equilibria, as well as computer simulations as toy models. The further works on this research inquiries would also be conjectured to the recognition of psychological aspects e.g. happiness or well-being-ness, to the emerging macro-pattern of economic decisions. This is left for future work.

\section{Acknowledgement}

Author thanks Surya Research International for the provision of financial support in which period the paper is written. All fault remains author's.

\section{Works Cited}

[1] Axelrod, R. (1997). "The Dissemination of Culture: A M odel with Local Convergence and Global Polarization". The Journal of Conflict Resolution 41 (2): 203-26. Sage.

[2] Chan, T. W. \& Goldthorpe, J. H. (2007). "The Social Stratification of Cultural Consumption: Some Policy Implications of a Research Project". Cultural Trends 16 (4): 373-84. Routledge.

[3] Friedman, D. (1998). "On Economic Applications of Evolutionary Game Theory". Journal of Evolutionary Economics 8: 15-43. Springer-Verlag.

[4] Friedman, D. \& Yellin, J. (2000). "Castles in Tuscany: The Dynamics of Rank Dependent Consumption". UC Santa Cruz Economics Working Paper No. 455.

[5] Friedman, D. \& Abraham, R. (2004). "Landscape Dynamics and Conspicuous Consumption". Paper presented at the 2004 Proceedings of the Society for Dynamic Games. URL: http://www.vismath.org/research/landscapedyn/articles/Tucson4.pdf

[6] Gilbert, N. \& Terna, P. (2000). "How to build and use agent-based models in social science". M ind \& Society 1 (1): 57-72. Springer. 
[7] Graham, C. \& Felton, A. (2006). "Inequality and Happiness: Insights from Latin America". Journal of Economic Inequality 4: 107-22. Springer.

[8] Hofbauer, J. \& Sigmund, K. "Evolutionary Game Dynamics". Bulletin of the American M athematical Society 40 (4): 479-519. AM S.

[9] Kahneman, D. (2003). Maps of bounded rationality: psychology for behavioral economics. The American Economic Review. 93(5). pp. 1449-1475. American Economic Association.

[10] Kirman, A., Livet, P., Teschl, M. (2010). "Rationality and Emotions". Philosophical Transactions of The Royal Society B 365: 215-9. The Royal Society.

[11] Macy, M.W., \& Willer, R. (2002). "From Factors to Actors: Computational Sociology and Agent Based Modeling. Annual Reviews Sociology 28: 143-66. Annual Reviews.

[12] Parravano, A., Rivera-Ramirez, H., \& Cosenza, M. G. (2006). "Intracultural Diversity in a M odel of Social Dynamics". Physica A 379 (1): 241-9. Elsevier.

[13] Plutchik, R. (1980). Emotion: A psycho-evolutionary synthesis. Harper \& Row.

[14] Situngkir, H. (2003). "M oneyscape: a generic agent-based model of corruption". BFI Working Paper Series WPD2003. Bandung Fe Institute.

[15] Situngkir, H. (2006). "Advertising in Duopoly Market". BFI Working Paper Series WPF2006. Bandung Fe Institute.

[16] Simon, H. (1990). "A mechanism for social selection and successful altruism". Science 250 (4988): 1665-8. AAAS.

[17] Smith, J. M. (1982) Evolution and the Theory of Games. Cambridge University Press

[18] Solomon, M., Bamossy, G., Askegaard, S., \& Hogg, M. K. (1999). Consumer Behavior: A European Perspective 3rd ed. Prentice Hall.

[19] Veblen, T. (1898). The Theory of the Leisure Class. Macmillan. 